\title{
Fumigation of Brown Rice with Volatiles from Bee Propolis and Impact on Storage Characteristics
}

\author{
Griffiths Atungulu $^{1 *}$, Shoji Koide ${ }^{2}$ and Shantae Wilson ${ }^{1}$ \\ ${ }^{1}$ Food Science Department, University of Arkansas Division of Agriculture, Fayetteville, 2650 N Young Avenue, Fayetteville, AR 72704, USA \\ ${ }^{2}$ Faculty of Agriculture, Iwate University, Morioka, 020-8550, Japan
}

\begin{abstract}
Received: September 09, 2014; Accepted: March 10, 2015; Published: March 20, 2015
*Corresponding author: Griffiths Atungulu, Food Science Department, University of Arkansas Division of Agriculture, Fayettevill 2650 N Young, Avenue, Fayetteville, AR 72704, USA, Tel : +1-530-304-1271; Email: atungulu@uark.edu
\end{abstract}

\begin{abstract}
Rice lipids are usually stable in the intact spherosomes in the cell. However, lipid membrane destruction by phospholipase, physical injury or high temperature, initiates hydrolysis through the action of lipases. Control of microbial induced lipolysis of rice would benefit from an approach that provides simultaneous antimicrobial and antioxidation control. Therefore, propolis, a resinous hive product collected by honeybees, known to possess both antimicrobial and antioxidant properties, was used in this study to evaluate feasibility of using its derived volatiles to control microbial prevalence, while providing oxidative stability of lipids, in stored brown rice (Oryza sativa, hinohikari var). Specifically, the effect of volatiles from Propolis including polar extracts (AEPE), non-polar extracts (MCPEV and HEPEV) and crude samples (CRUPV), on rice lipids oxidation and fungal and bacterial prevalence were studied. The stabilization effectiveness on oleic, linoleic and palmitic acids in brown rice were CRUPV > AEPEV > MCPEV $>\mathrm{HEPEV}>$ control, in the order of strength. Following brown rice storage, the bacterial species of Bacillus genera were most predominant with Bacillus cereus persisting on samples of all the treatments. Moraxella osloensis, Pseudomonas oleovorans, Sphingomonas paucimobilis, Xanthomonas campestris, Curtobacterium citreum, Curtobacterium flaccumfaciens and Aerococcus viridans affected the samples discriminately. Penicillium species was the most dominant fungi and persisted in all the treatments. MCPEV and CRUPV treated samples indicated the lowest species infestation numbers. Milling of the treated and stored brown rice samples indicated that samples treated with MCPEV and CRUPV had less broken rice than control samples. Also, the MCPEV treated samples were whiter than the control samples. The findings from this study show that there is potential to use Propolis extracts to limit lipid oxidation and microbial prevalence on rice.
\end{abstract}

Keywords: Rice lipids; Microorganism; Propolis

\section{Introduction}

Rice lipids, which consists mainly of triacylglycerols and occur in the spherosomes located in the aleurone cells and embryo, are mainly responsible for the deterioration of rice flavor. Oxidation of the lipids degrades the organoleptic quality of the product, reduces its nutritional value, and products of the oxidation processes can participate in the aging of an organism and in the aetiology of cardiovascular diseases and cancer
[1]. Lipids can be protected against uncontrolled oxidation by addition of antioxidants with the ability to remove the free radicals and Reactive Oxygen Species (ROS) that damage cellular and tissue structures. There is currently much interest in natural antioxidants, including polyphenols, isolated from plants [2]. Because of their natural occurrence and consumption with plants, they are fully accepted by consumers, contrary to synthetic antioxidants which may initiate disease. Much research is therefore being conducted to find completely safe, naturally active substances that strongly inhibit degradation of lipids in food systems. Lipolytic behavior of some microorganisms associated with rice storage demand that any such antioxidant should provide antimicrobial and antioxidative activities simultaneously.

Propolis, a natural product that is recently gaining application in pharmacology as well as great attention in the food industries, is a resinous hive product collected by honeybees from parts of plants, buds and exudates, and has been used as a folk medicine since around $300 \mathrm{BC}$. It has been shown to possess antiinflammatory, immunostimulatory, antiviral and antibacterial activities [3]. The pharmacological properties of propolis are principally attributed to the presence of flavonoids. These are reported to have anti-inflammatory, antihepatotoxic, anticancer and antiviral activities, as well as antibacterial effects. The prevailing view is that the broad biological activities of flavonoids are related, at least in part, to their ability to protect against the damaging action of free radicals. Extract of the culinary herb rosemary (Rosmarinus officinalis), used as a positive control in this experiment, is also known to contain materials with antioxidant properties and is currently used in Japan as a commercial antioxidant.

In our previous research work we explored the application of steam distilled extracts of propolis [4] and reported the antilipid peroxidative action of the extracts in the static gas phase on rice bran. Whereas those findings were only limited to the propolis portion that could be steam distillated and with associated very low recovery of essential oils, it was obvious from tests then that 
the remaining portion could still indicate activity. Our subsequent work [5] therefore endeavored to study the effect of modifying storage environment by using propolis vapor obtained through a rather non-thermal mode of extraction (solvent fractionation). However, the findings reported remained non conclusive with regard to the effect of the treatments on the individual components of the fatty acids as well as the genotype analysis of bacteria, fungi and their prevalence on treated samples. Consequently, we endeavored to comprehensively investigate the effect of the treatment on the major fatty acid components of rice lipids (oleic, linoleic and palmitic acids) and further elucidate the bacterial and fungal distribution on the treated product by genotype identification of isolated bacteria through genotype analysis techniques of 16s ribosomal DNA sequence and microscopic observation of fungal morphological features respectively. Since the brown rice is to be milled after storage the impact of these treatment on the milled rice whiteness and head rice were also studied. It is hypothesized that the diffusion of the volatiles into the stored product varied under natural diffusion and could affect the bran layer of the brown rice and the whole kernel variedly in terms of lipid stabilization. Polar volatiles and two non-polar fractions were utilized in our experiments.

\section{Materials and Methods \\ Propolis and extract of the culinary herb rosemary}

2.2. In this experiment crude propolis harvested at Minas Gerais in Brazil was used. The propolis was synthesized by Apis mellifera bees from a botanical source that was predominantly of Baccharis dracunculifolia tree species. The propolis sample used was supplied by Yamada Apiculture Center, Inc., Japan. Crude propolis in this article refers to the solid, non-fractionated propolis as obtained from the apicultural center. Rosemary oilsoluble (RM21Bbase) and water-soluble (RM21Abase) extracts (Mitsubishi Kagaku Foods Corp., Japan) were used as positive controls. The procedure for the preparation of the two rosemary

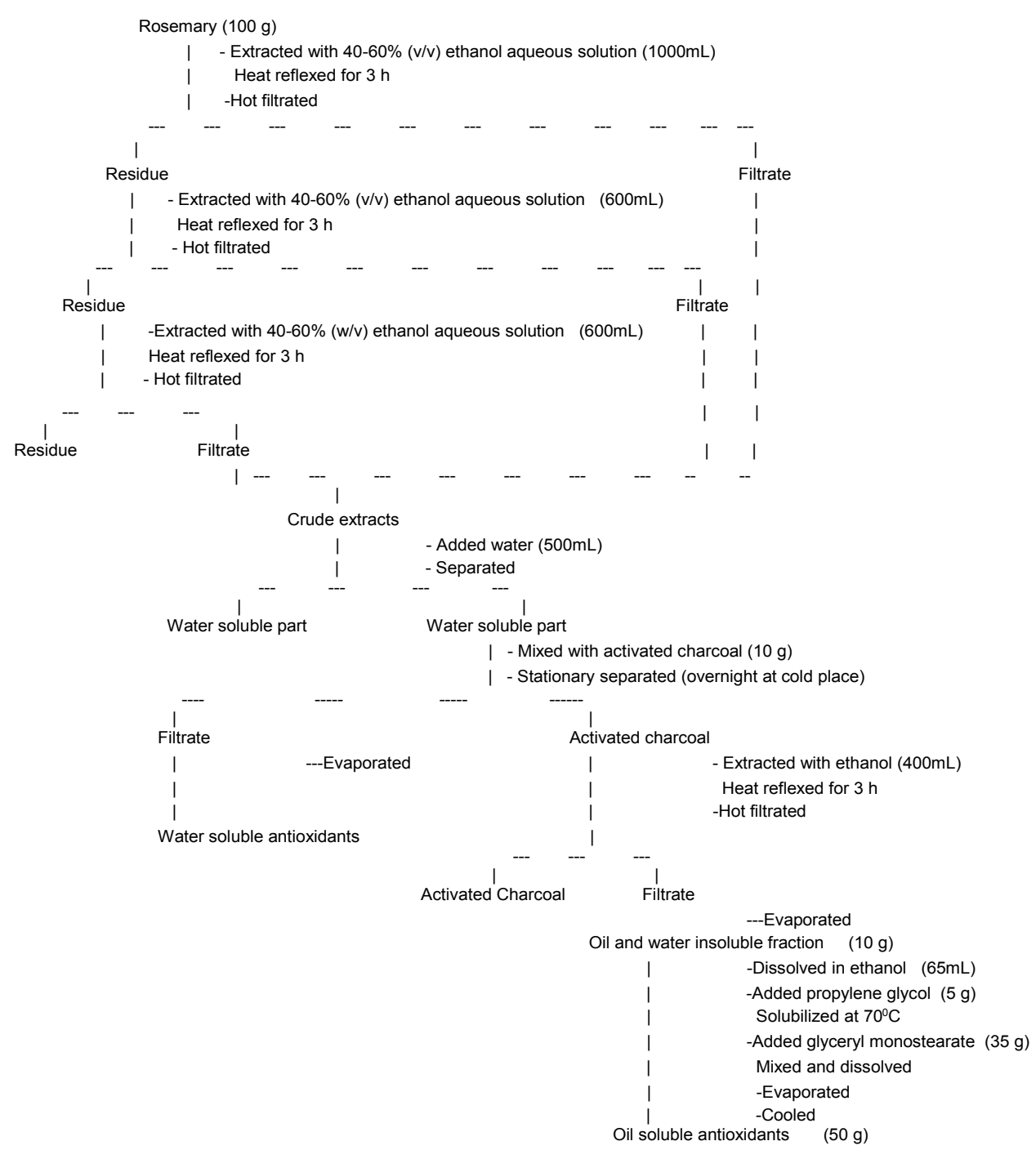

Table 1: Preparation procedure of antioxidant from rosemary (RM21 A base and RM 21 Bbase supplied from Mitsubishi-kagaku Foods Corporation) according to the patented method of Tokyo Tanabe Seiyaku Co. Ltd presently Mitsubishi Pharmaceutical Corporation. 
extracts is shown in Table 1. Unless otherwise stated, all chemicals used were of analytical grade and were obtained from or through Wako Pure Chemicals Co., Ltd., Japan.

\section{Fractionation of the propolis sample}

To obtain polar and non-polar fractions, the crude propolis was fractionated using solvent extraction. The polar fraction was obtained by using absolute ethanol extraction (AEPE) while other two non-polar fractions were extracted using methylene chloride (MCPE) and hexane (HEPE). The sub fractions are herein henceforth referred to as AEPE, MCPE, and HEPE when in liquid form and AEPEV, MCPEV, and HEPEV respectively when referring to their volatile forms. Extraction was performed according to Silici and Koc [6] with some modification. In extraction process, a $30 \mathrm{~g}$ sample of the crude propolis was mixed with $100 \mathrm{ml}$ of solvent (ethanol, hexane or methylene chloride) and kept on a continuously running shaker (120 rpm) for seven days at $25^{\circ} \mathrm{C}$. After the seven days, filtration was performed and the filtrated product was centrifuged (1000 rpm, $5 \mathrm{~min}$ ) to remove fine particles from the filtrate. This was followed by rotary evaporation of the filtrate under vacuum to concentrate the isolated sub-fractions and expel the solvent. The sample was then flushed with nitrogen to drive out the solvent completely. The weight of material recovered was weighed for yield computation and obtained as follows: $0.30 \pm 0.07 \mathrm{~g}, 0.41 \pm 0.01 \mathrm{~g}$ and $0.05 \mathrm{~g}$ of extract per $\mathrm{ml}$ of methylene chloride, absolute ethanol and hexane, respectively. These samples were kept at $4^{\circ} \mathrm{C}$ until use.

\section{Qualitative characterization of the propolis sample}

Details of the methods employed in qualitative characterization of the Propolis samples such as those of using DPPH free radical assay and GC/MS are described by Atungulu et al. [5]. In this study, selected ion monitoring (SIM) mode was employed during the GC/MS analysis. The standards were pure chemicals obtained from Wako chemical Co., Japan (caffeic acid, ferulic acid, Caffeic Acid Phenethyl Ester (CAPE)).

\section{Infusion of propolis in synthetic adsorbents}

Synthetic adsorbents (Sepabeads ${ }^{\circledR}$ SP825, Mitsubishi Chemical Co., Japan) were used to carry the propolis extracts. The synthetic absorbent was of the aromatic type based on cross-linked polystyrenic matrix with characteristics as follows: apparent density 670 g/L-R, moisture content 57\% (w.b.), pore volume $1.4 \mathrm{ml} / \mathrm{g}$, surface area $1000 \mathrm{~m}^{2} / \mathrm{g}$ and pore radius $57 \mathrm{~A}^{\circ}$. The fractionated propolis extracts were infused into the synthetic adsorbent by firstly diluting a known weight of the sample with its corresponding solvent before mixing with the synthetic adsorbent. On absorbing the samples, the resulting slurry-like substance containing the active propolis sample was placed under a hood and flushed with nitrogen gas until weight change with time was negligible. This ensured the solvent was completely removed before use in experiments. In our experiment up to $48 \mathrm{~h}$ exposure ensured complete elimination of the extracting solvents for the adsorbent to solvent/sample mass ratio used.

\section{Brown rice and lipid analyses}

Brown rice used in this experiment was obtained from Kyushu University Farm, Fukuoka Prefecture in Japan. Post harvest treatment involved drying the grains to moisture content of $15.7 \%$ (w.b.) after which they were stored at $4^{\circ} \mathrm{C}$ until use. Damaged rice was removed by a rice quality inspector RGQI2A (Satake Corp., Japan). During treatment, a $1.5 \mathrm{~kg}$ of sample of brown rice was kept in a sterile and porous polyethylene net and placed inside an air tight desiccator that had the synthetic adsorbent infused with propolis extracts (AEPE, HEPE, MCPE) placed inside and at the bottom, such that volatiles from the adsorbents moved upwards to affect the stored sample. The controls were as rice placed inside dessicators with absorbent inside the desiccator's void of propolis extracts. The desiccators with products inside were kept in thermostatic chambers set at $30^{\circ} \mathrm{C}$. After the treatment the brown rice was milled using a Magic Mill SKM-5A (Satake Corp., Japan).

\section{Lipid extraction}

Lipid extraction was carried out according to a modified version of Miura, Yamauchi, Ogawa and Shibasaki [7] method. Lipids were extracted from the brown rice using chloroformmethanol $(2: 1, \mathrm{v} / \mathrm{v})$. Distilled water $(16 \mathrm{ml})$ was added to a $5 \mathrm{~g}$ product sample and the mixture was homogenized. The sample was brought in the separating funnel and $100 \mathrm{ml}$ chloroformmethanol solution was added. A 5 min shaking extraction was performed and the water layer and chloroform layers were separated. Successive two times shaking extractions were conducted on the water layer each time using $100 \mathrm{ml}$ chloroformmethanol $(2: 1, \mathrm{v} / \mathrm{v})$. The collected chloroform layer was combined and cleaned with $100 \mathrm{ml}$ of $0.5 \%$ Sodium Hydroxide $(\mathrm{NaOH})$ and then dehydrated with anhydrous sodium sulfate (adequate dose, $5 \mathrm{~g}$ in our experiment). The sample was then filtered and then followed by rotary evaporation of the filtrate under vacuum at the lowest temperature setting (below $40^{\circ} \mathrm{C}$ ). The vacuum during rotary evaporation was broken under nitrogen to avoid oxidation of the sample. Finally, all the solvent was expelled by nitrogen flushing at below $40^{\circ} \mathrm{C}$.

\section{Preparation of methylated fatty acid}

Fat extract (20 mg) was dissolved in $1 \mathrm{ml}$ benzene and 2 $\mathrm{ml}$ of sodium methoxide $(0.5 \mathrm{~N})$ was added. The mixture was shaken and left for 10 minutes at room temperature and then $0.5 \mathrm{~N}$ acetic acid aqueous solution was added for neutralization. This was followed by one minute shaking extraction using $5 \mathrm{ml}$ of hexane. The water and hexane layer were separated and the water layer once again subjected to a further 1 minute shaking extraction using $5 \mathrm{ml}$ hexane. A mixture of sodium sulfate and sodium hydrogen carbonate $(2: 1 \mathrm{w} / \mathrm{w})$ was added to the hexane layer and after filtration the solvent was expelled by nitrogen flushing below $40^{\circ} \mathrm{C}$. The solid was dissolved in $5 \mathrm{ml}$ hexane and the mixture subjected to GC analysis.

\section{Gas chromatography (GC) analysis of fatty acids}

GC analysis was performed after preparing methylated fatty acids of the fat extract from the treated sample to determine 
the profiles of oleic, linoleic and palmitic acids. A GC-4000 (GL Sciences Inc.) equipped with an Inert Cap 225 capillary column (0.25 mm i.d. $\times 30 \mathrm{~m}$ length, $0.25 \mu \mathrm{m}$ df, GL Sciences Inc., Japan) was used. The oven, injection, detector (FID) temperatures were $210^{\circ} \mathrm{C}, 230^{\circ} \mathrm{C}$ and $230^{\circ} \mathrm{C}$ respectively. Helium gas at a flow rate of $1.33 \mathrm{ml} / \mathrm{min}$ and split mode of injection (1:100) were employed. Methyl esters of the fatty acid standard chemicals (linoleic, oleic and palmitic acids) were obtained from Wako Chemical Co., Japan. All reagents were of analytical grade, and distilled water was used throughout.

\section{Microbial identification}

Fungi: Following treatment, isolation and identification of storage-related fungi was performed. The fungi were distinguished visually based on morphological features and by microscopic observation.

Bacteria: Three times pure-culturing which involved a four step procedure of enrichment, plating, stock culturing and characterization of bacteria was performed. The bacteria were visually identified using morphological features (shape, size, color, elevation, margin type) and confirmed by DNA genotype identification as follows. Each bacterial pure culture was picked using a colony inoculating loop and placed in 0.2-0.5 ml tubes containing $80 \mu \mathrm{L}$ of Prep Man Ultra Sample Preparation Reagent (DNA extraction kit, Applied Biosystems). The tubes were covered and thoroughly mixed using a vortex (ASONE Co. Japan). A tabletop centrifuge (CHIBITAN, Japan) was used after mixing to ensure the mixture settles at the bottom of the tubes. Heat treatment $\left(99.9^{\circ} \mathrm{C}\right.$ for $10 \mathrm{~min}, 10^{\circ} \mathrm{C}$ for $3 \mathrm{~min}$ and then $4^{\circ} \mathrm{C}$ constant) was performed using a thermo-recycler (Program Temperature Control System, ASTEC, Fukuoka Japan). The sample was then centrifuged (5922, KUBOTA, Japan) at 15000rpm for 3 min. The supernatant was removed and put in separate tubes while the bacterial debris was disposed.

Standard PCR reagents and treatments were used. A sample of $12 \mu \mathrm{l}$ of PCR reagent was dispensed per $1 \mu \mathrm{l}$ of DNA template. The PCR master mix included Taq DNA polymerase, PCR buffer, dNTPs mixture (TaKaRa Bio Co., Japan, primers (Forward/ Reverse, Sigma Aldrich Co., USA) and ultra pure water. The mixture quantity was $1 \mu \mathrm{L}$ of DNA template (our extracted sample), 0.2 $\mu \mathrm{l}$ of Taq DNA polymerase $\left(\mathrm{Mg}^{2+}\right), 1.25 \mu \mathrm{l}$ of PCR buffer, $1.25 \mu \mathrm{L}$ of dNTPs mixture, $0.5 \mu \mathrm{L}$ of Forward primer, $0.5 \mu \mathrm{L}$ of Reverse primer and $8.3 \mu \mathrm{L}$ of ultra pure water. The programmed thermorecycling for the PCR run was 1 cycle $\left(94^{\circ} \mathrm{C}\right.$ at $\left.3 \mathrm{~min}\right)$, followed by 30 set cycles $\left(94^{\circ} \mathrm{C}\right.$ for $30 \mathrm{sec}, 55^{\circ} \mathrm{C}$ for $30 \mathrm{sec}$ and $72^{\circ} \mathrm{C}$ for $\left.1 \mathrm{~min}\right)$ then $72^{\circ} \mathrm{C}$ for $7 \mathrm{~min}$ and then stabilize at $4^{\circ} \mathrm{C}$.

Agarose gel (1\%) was prepared. The agarose powder was dissolved into Tris/Acetic Acid/EDTA Buffer (TAE, Bio-Rad laboratories, USA CA) and paper-wrapped to avoid contamination. Microwave heating was used to facilitate complete dissolution of the agar powder into the buffer. The clear solution was removed, allowed to cool and then transferred into the gel tray. About 2-3 $\mu \mathrm{L}$ of PCR product was mixed with loading buffer Blue Juice Gel (Wako Pure Chemical Co., Japan): 1\% Bromophenol blue (BPB), 1\% Xylene cyanol FF (XC), 500 mM EDTA, glucose and ultra pure water. The parafilm pipetting method was used for mixing. On the first leftmost hole of the sample wells in the electrophoresis chamber (Genius and S-PitSub Ks-8429, Japan), $5 \mu$ DNA molecular marker (100bp Ladder Marker, TaKaRa Bio Inc., Shiga, Japan) able to confirm the 20bp-1500bp range was put. Electrophoresis buffer, Tris-acetate-EDTA (TAE) was used. The fluorescent dye, ethidium bromide solution $(100 \mu \mathrm{L} / 1$ liter of ultra pure water) was used for staining the gel. A 254-nm UV Transilluminator (ATTO Bioinstrument, Tokyo, Japan) was used to visualize ethidium bromide-stained DNA in gels.

The following procedure was undertaken to purify amplified PCR products and perform DNA sequencing: For purification purposes, $2 \mu \mathrm{L}$ of amplified PCR product was put in tubes and $0.8 \mu \mathrm{L}$ of ExoSAP-IT (GE healthcare UK Ltd., Amersham Place, England) added. The following thermal treatment (Program Temperature Control System, PC708, ASTEC, and Fukuoka Japan) was then performed: $37^{\circ} \mathrm{C} 15$ minutes incubation and $80^{\circ} \mathrm{C} 15$ minutes heating. For DNA Sequencing, the mixture for sequencing reaction was first prepared. The ABI Prism $₫$ BigDye ${ }^{\circledR}$ terminator v.3.1 cycle sequencing kit (Applied Biosystems, CA USA) was used. The reaction mixture was $1.0 \mu \mathrm{L}$ template (purified PCR product), $0.25 \mu \mathrm{L}$ Primer (Reverse), $1.0 \mu \mathrm{L}$ Sequencing buffer, $0.5 \mu \mathrm{L}$ BigDye and $2.25 \mathrm{ml}$ Ultra pure water. Thermal-recycling (Program Temperature Control System, PC708, ASTEC, Fukuoka Japan) of the mixture was performed in serial cycles thus: 1 cycle $\left(96^{\circ} \mathrm{C}\right.$ for $\left.60 \mathrm{sec}\right), 25 \mathrm{cycles}\left(96^{\circ} \mathrm{C}\right.$ for $10 \mathrm{sec}, 50^{\circ} \mathrm{C}$ for $5 \mathrm{sec}, 60^{\circ} \mathrm{C}$ for 4 min was) and final kept at $4^{\circ} \mathrm{C}$ constant.

Ethanol Precipitation was done by mixing $5.0 \mu \mathrm{L}$ of the sample (after the sequencing reaction) with $5 \mu \mathrm{L}$ ultrapure water, $1 \mu \mathrm{L}$ of $3 \mathrm{M}$ Sodium acetate $\left(\mathrm{CH}_{3} \mathrm{COONa}\right)$ and $25.0 \mu \mathrm{L}$ of $95 \%$ ethanol. The mixture was left for $15 \mathrm{~min}$ at room temperature and then centrifuged (5922, KUBOTA, Japan) at 15000 rpm for $20 \mathrm{~min}$. Ethanol was removed using a syringe; care being taken not to touch the surface of the tubes. Then $200 \mu \mathrm{L}$ of $70 \%$ ethanol was added and again centrifugation performed at $15000 \mathrm{rpm}$ for $5 \mathrm{~min}$. The ethanol was perfectly removed and the tubes left to dry completely. Genetic analysis grade $20 \mu \mathrm{L}$ sample of $\mathrm{Hi}-\mathrm{Di}^{\mathrm{TM}}$ Formamide (Applied biosystems, Foster CA) was added and then vortexed for $20-30$ seconds. The sample was subjected to heat shock treatment thus: $95^{\circ} \mathrm{C}$ for $3 \mathrm{~min}$ and then immediately cooled in ice at $0^{\circ} \mathrm{C}$ for $5 \mathrm{~min}$. The product was then transferred into DNA sequencer compatible tubes. Care was taken not to allow any bubbles in the solution. The tubes were sealed with rubber seals and covered with aluminum foil until loading on sequencer so as to avoid irradiation with light. DNA Sequencing was performed using an ABI Prism 3100-Avant genetic analyzer (Applied Biosystems). The sequencing data was uploaded to DDBJ (DNA Data Bank of Japan) website (http://www.ddbj.nig. ac.jp/), and the database comparison (BLAST) were conducted whereby the closest matches with bacterial genus and species were obtained.

\section{Post treatment quality analysis}

The post treatment milled rice quality indices including percentage weight of whole kernel relative to initial weight of 
brown rice (apparent head rice), cumulative rice damage, and rice whiteness were determined using a rice quality inspector RGQI-2A (Satake Corp., Japan). Cumulative damaged rice lumped together any broken, malformed, dead, colored or cracked grain. Immature grain had initially been removed prior to storage experiments.

\section{Results and Discussion}

\section{Lipid analysis}

Propolis samples used in this experiment were similar to the ones previously used and reported in our previous experiments [5]. Detailed results on qualitative characterization of the propolis samples used in this article involving the analysis of the scavenging activity on DPPH free radical, antioxidant activity analysis through the $\beta$-carotene-linoleic acid system of assay [8] and the gas chromatograph mass spectrometer (GC/MS) qualitative analysis can therefore be obtained in our previous report [5].

The changes occurring in the three major fatty acids related to rice were studied and their levels in the whole grain investigated during a 12 months storage period under various treatments with crude propolis (CRUPV) and its extracts (AEPEV, MCPEV, and HEPEV). Figure 1 shows the respective compositional changes of oleic, linoleic and palmitic acids in brown rice following the treatments.

It was clear from the results that oleic acid concentration was the highest followed by linoleic and palmitic acids. The CRUPV treated brow rice maintained the highest amount of all the three fatty acids. It was observed that with reference to the initial amounts of these fatty acids, AEPEV, MCPEV and HEPEV, in the respective order of strength, stabilized the fatty acids during the studied storage period. The degradation of these fatty acids was therefore affected by the treatments.
Rice lipids, which consists of mainly triacylglycerols, occur in the spherosomes located in the aleurone cells and embryo, and are responsible for deterioration of rice flavor. Many investigations about stale flavor of rice have been carried out. Yasumatsu, Moritaka and Wada $[9,10]$ have reported that free fatty acids were released from neutral lipids during storage and that the increase in their amount resulted in deterioration of rice flavor. The composition ratios reported in this study are within the ranges cited by other reports $[11,12]$.

In spite of intensive effort by many investigators, the detailed degradative process of the rice lipids remains obscure, especially the trigger of the degradation, during storage. In this study, the mechanism through which propolis derivatives affected the lipids is not clear.

Based on previous findings [5], the propolis extracts used showed free radical scavenging activity in a concentrationdependent manner. The extract concentration providing $50 \%$ inhibition $\left(\mathrm{IC}_{50}\right)$ of DPPH free radical were 9.8, 3.2, $2.8 \mu \mathrm{g} / \mu \mathrm{L}$ for HEPE,AEPE and MCPE respectively. RM-21Abase and RM-21Bbase rosemary extracts had $\mathrm{IC}_{50}$ values of $1.9,1.8 \mu \mathrm{g} / \mu \mathrm{L}$ respectively. Furthermore, the $\beta$-carotene bleaching assay clarified that AEPE was most the effective of the propolis derivative [5]. The study reported that the oxidative degradation rates of $\beta$-carotene/ linoleic acid emulsion monitored spectrometrically at $50^{\circ} \mathrm{C}$ were lowest for AEPE and highest for HEPE at $4.3 \times 10^{-4} \mathrm{~min}^{-1}$ and 1.9 $\mathrm{x} 10^{-3} \mathrm{~min}^{-1}$, respectively.

New information revealed in this research included GC/MS quantitative analyses employing selected ion monitoring (SIM) mode whereby the caffeic acid, ferulic acid, caffeic acid phenethyl ester (CAPE) were found dominat in the extracted propolis. Although only the three components (caffeic acid, CAPE, and ferulic acid) were identified, a myriad of other compounds were still prevalent in the three derivatives according to the TIC results

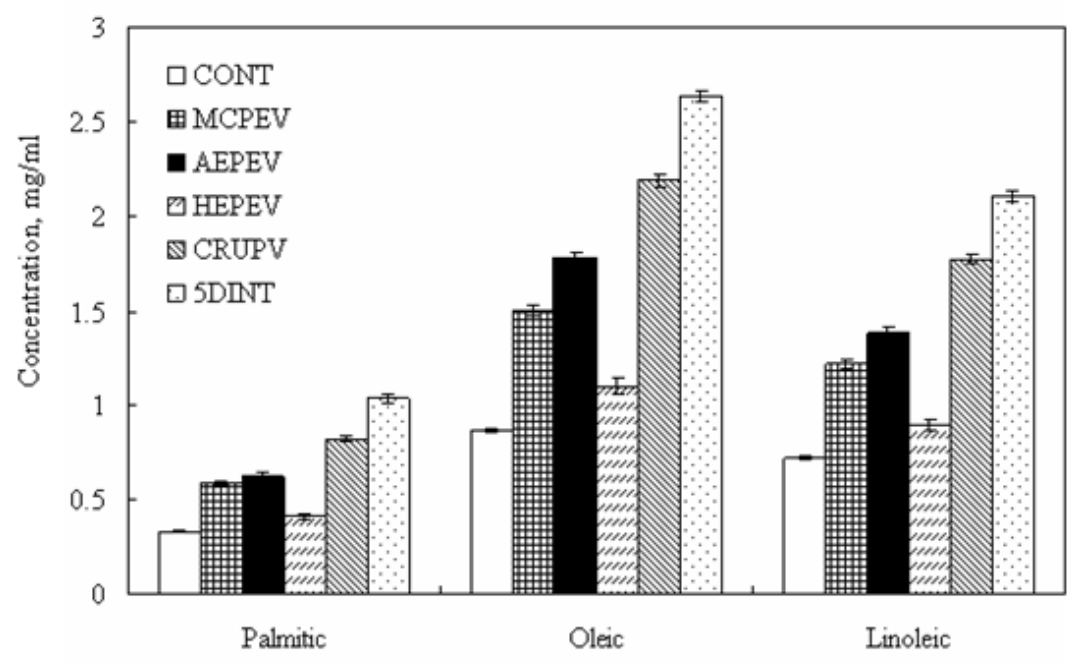

Figure 1: Fatty acids stabilization using polar and non-polar fractions of propolis after twelve months at room conditions (30 $\left.{ }^{\circ} \mathrm{C}\right)$ : $\mathrm{MCPEV}, \mathrm{AEPEV}$, and HEPEV are sub fractions prepared by methylene chloride, absolute ethanol, and hexane extraction respectively; CONT, CRUPV, and 5DINT are samples at control and crude propolis treatments, and initial conditions respectively. 


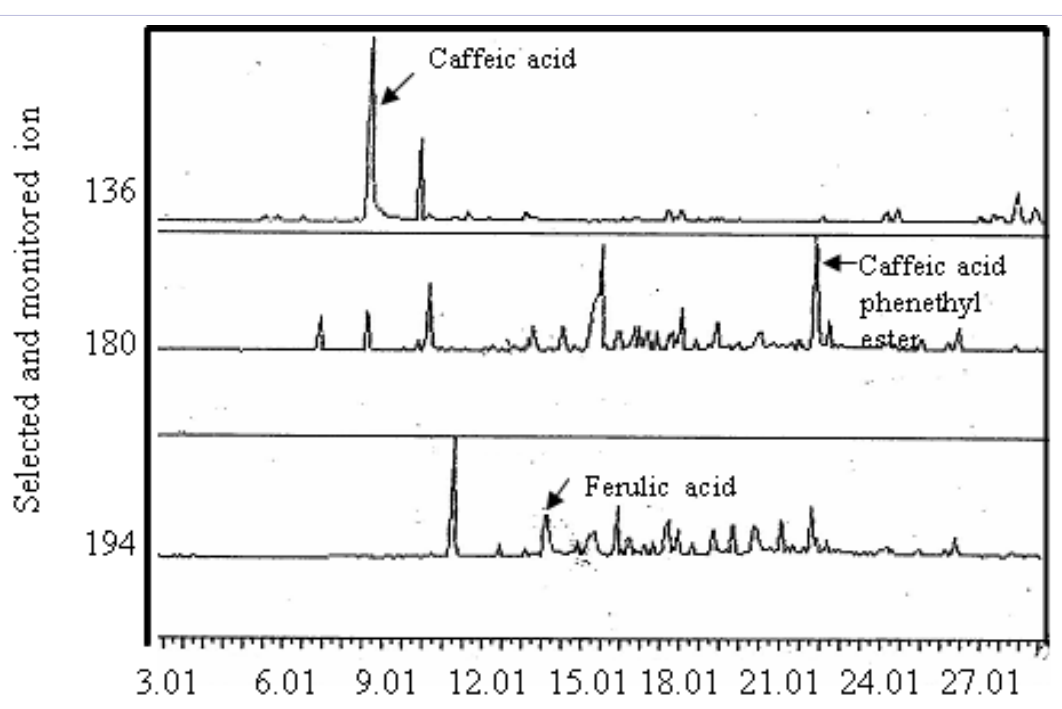

Retention time, min

Figure 2: Typical GC_MS/SIM obtained by selected ion monitoring (SIM) for absolute ethanol prepared propolis extract (AEPE). Caffeic acid retention time $(\mathrm{RT})=8.64$, molecular Weight $(\mathrm{MW})=180$; Caffeic acid phenethyl ester, RT = 23.5, MW = 284 and Ferilic acid RT $=14.5, \mathrm{MW}=194$.

(Figure 2). The identified compounds and perhaps others in the extracts may be associated with the antioxidative effects leading to inhibiting oxidation of oleic, linoleic and palmitic acids.

\section{Microbial analysis}

Table 2 illustrates the distribution of bacteria that thrived on brown rice samples treated with extracts processed from propolis. Bacteria of the order Bacillales, Burkholderiales, Enterobacteriales, Pseudomonadales, Sphingomonadales, Rhizobiales, Xanthomonadales, Antinomycetales and Lactobacillales were found in the samples, albeit discriminately and prevalence depended on the treatments. The treatment 5DINT shows the bacterial distribution as detected in the samples before any treatment. The samples were mixed to improve the uniformity of bacterial distribution. In spite of this we noted that some bacteria were not detectable in the initial sample. These were notably from the spore forming bacterial orders of the Bacillales, Antinomycetales, Lactobacillales with an exception of the gram negative Moraxella osloensis. The initial sample (5DINT) had been previously stored at $5^{\circ} \mathrm{C}$. It was noted from the results that each treatment presented different conditions and no single treatment could eliminate all the bacteria initially detected in the samples. Thus, in pursuant to industrial application, the synergistic effect of each derivative would have to be tapped into for optimizing the sterilization effect from propolis. Evidently, Bacillus cereus persistently occurred in all the treatments indicating its susceptibility to prevail in the grain treated with the propolis extract dosage applied in this experiment.

Table 3 illustrates the distribution of fungi that thrived on the rough rice after various treatments. In the initial sample (5DINT) hardly any fungi were detected. However, depending on the treatment, fungi of the following genera were detected in the brown rice after 12 months of storage: Aspergillus, Absidia,
Penicillium and Talaromyces. MCPEV treatment gave the least number of thriving fungal species followed by the CRUPV treatment. Penicillium species was the most dominant fungi and persisted in all the treatments.

\section{Synergistic effects of propolis extracts on bacteria and fungi prevalence}

There were some synergisms among the propolis treatments on bacterial and fungal prevalence on treated rice. The implications of the treatments versus bacteria prevalence in the initial samples (5DINT) could explain the synergism of the propolis treatments. Based on the results, a combination of treatments would ensure complete elimination of some bacterial microbes. For instance, a combination of AEPEV, MCPEV, and HEPEV would eliminate all the bacterial species identified in Table 2, except Bacillus cereus. It was also observed that bacteria in the order of Burkholderiales were prevalent in the initial sample but diminished when subjected to the propolis treatments. It is, however, not conclusive whether the inactivation of these particular bacteria was purely due to the effect of the propolis extract since the microbe was also not detected on the control samples.

Fungi of the genus Aspergillus, Absidia, Penicillium, and Talaromyces were prevalent of the rice samples. It was observed from results that a combination of AEPEV, MCPEV, and HEPEV treatments would eliminate most of the fungi on rice but one species each of Penicillium and Talaromyces molds. Using MCPEV alone gave the best result of fungal inactivation. Of the molds identified in the study, only molds of the genus Penicillium remained resistant to MCPEV.

\section{Effects of treatments on rice quality}

After storing brown rice samples for 12 months under various treatments, the samples were milled and separated into broken 
Table 2: Bacterial distribution on brown rice following various treatment as identified by genotype analysis. MCPEV, AEPEV, and HEPEV are sub fractions prepared by methylene chloride, absolute ethanol, and hexane extraction respectively; CONT, CRUPV, and 5DINT are samples at control and crude propolis treatments, and initial conditions respectively. The symbol "o" means the corresponding microorganism was observed.

\begin{tabular}{|c|c|c|c|c|c|c|c|}
\hline \multicolumn{2}{|c|}{ Bacterial distribution } & \multicolumn{6}{|c|}{ Treatment } \\
\hline Order & Genus species & AEPEV & MCPEV & HEPEV & CONT & CRUP & 5DaTT \\
\hline \multirow[t]{9}{*}{ Bacillales } & Bacillus pumilus & - & • & & • & • & \\
\hline & Bacillus cereus & - & • & - & - & - & \\
\hline & Paenibacillus amyloyticus & - & & & - & & \\
\hline & Paenibacillus polymyxa & & • & & • & & • \\
\hline & Bacillus coahuilensis & & & - & & & \\
\hline & Bacillus thuringiensis & & & - & & & \\
\hline & Bacillus subtilis & & & & $\bullet$ & & \\
\hline & Bacillus atrophaeus & & & & - & & \\
\hline & Paenibacillus barcinonensis & & & & & $\bullet$ & \\
\hline Burkholderiales & Burkhokleria gladioli & & & & & & • \\
\hline \multirow[t]{2}{*}{ Enterobacteriales } & Pantoea agglomerans & & & & & & • \\
\hline & Pantoea ananatis & & & & & & • \\
\hline \multirow[t]{3}{*}{ Pseudomonadales } & Moraxella osloensis & • & & - & & & \\
\hline & Pseudomonas oleovorans & & - & & & & - \\
\hline & Pseudomonas oryzihabitans & & & & & & $\bullet$ \\
\hline zSphingomonadales & Sphingomonas paucimobilis & & • & & • & • & • \\
\hline \multirow[t]{3}{*}{ Rhizobiales } & Agrobacterium tumefaciens & & & & & & • \\
\hline & Methylobacterium aquiticum & & & & & & - \\
\hline & Methybacteriumfujisawaense & & & & & & • \\
\hline \multirow[t]{2}{*}{$\mathrm{X}$ anthomonadales } & Xanthomonas campestris & & - & & & & - \\
\hline & Stenotrophomonas maltophilia & & & & & & $\bullet$ \\
\hline \multirow[t]{5}{*}{ Antinomycetales } & Curtobacterium citreum & & • & & & & • \\
\hline & Curtobacteriumilaccumfaciens & & • & & & & \\
\hline & Microbacteriwn kitamiense & & & & & & • \\
\hline & Microbacteriumphyllosphaerae & & & & & & • \\
\hline & kficrobacterium oxydans & & & & & & - \\
\hline Lactobacillales & Aerococcus viridans & & • & & & & \\
\hline
\end{tabular}

Table 3: Fungal distribution: identification of genus by microscopic observation of the species-specific differentiated structures.

\begin{tabular}{|l|c|c|c|c|c|}
\hline Fungal distribution & \multicolumn{5}{|c|}{ Treatment and species infestation number } \\
\hline Genus & AEPEV & MCPEV & HEPEV & CONT & CRUPV \\
\hline Aspergillas & ND & ND & ND & ND & 1 \\
\hline Absidia & 1 & ND & 5 & ND & ND \\
\hline Penicillium & 6 & 1 & 7 & 8 & 3 \\
\hline Talaromyces & ND & ND & 1 & ND & ND \\
\hline
\end{tabular}

and non-broken (3/4 kernel size and longer). The percentage of the original sample that remained as non-broken was evaluated. The overall non-broken rice percentage in all the treatments was higher than $70 \%$ (Figure 3).

The percentage of brown rice that remained non-broken after milling declined in all the cases except for MCPEV treatment which retained the same percentage value as in the initial sample.
AEPEV and HEPEV indicated lower percentage of non-broken rice values compared to the control. Similarly, MCPEV provided the highest whiteness value for the processed rice. The cumulative damage in this experiment included dead, malformed, colored, cracked and broken grains. From visual observation, AEPEV and HEPEV treated grains appeared slightly off color; this was also noted from the lower whiteness values as indicated in Figure 3. 


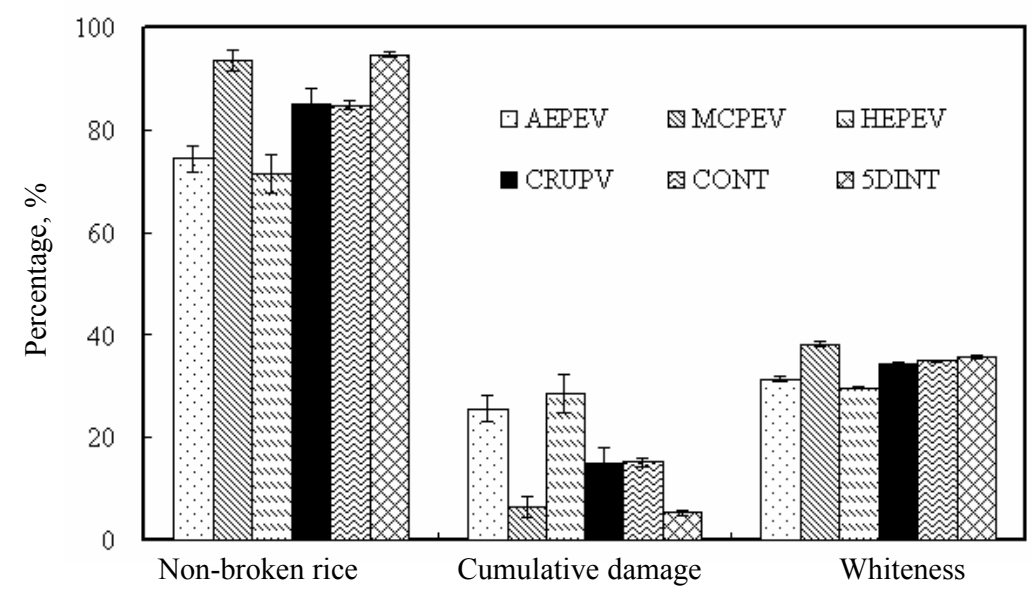

Figure 3: Effects of treatments on the quality of rice milled after treatment, control and initial conditions: MCPEV, AEPEV, and HEPEV are sub fractions prepared by methylene chloride, absolute ethanol, and hexane extraction respectively; CONT, CRUPV, and 5DINT are samples at control and crude propolis treatments, and initial conditions respectively.

Because of this discoloration, the corresponding score in terms of cumulative damage was also higher. Conversely, grain treated with MCPEV had higher whiteness score and this corresponded to the lowest cumulative damage almost same as that of the initial sample.

\section{Conclusions}

The effect of fumigating brown rice with volatiles from polar (AEPE) and non-polar (MCPEV and HEPEV) extracts and crude (CRUPV) of propolis on storage characteristics including lipid degradation, fungal and bacterial prevalence, and milling quality was evaluated. The treatments affected the composition of oleic, linoleic and palmitic acid during storage. The CRUPV treated brow rice maintained the highest amount of all the three fatty acids. With reference to the initial amounts of the fatty acids, AEPEV, MCPEV and HEPEV, in the respective order of strength, stabilized the fatty acids during storage. Genera Bacillus was the most dorminant bacteria on stored brown rice with Bacillus cereus persistently present on all treated samples. Moraxella osloensis, Pseudomonas oleovorans, Sphingomonas paucimobilis, Xanthomonas campestris, Curtobacterium citreum, Curtobacterium flaccumfaciens and Aerococcus viridans were found present on stored brown rice but occurred discriminately. No fungi were detected on initial brown rice samples, but after 12 months of storage, fungi of Aspergillus, Absidia, Penicillium and Talaromyces species were present and depended on the treatment. Fungi of Penicillium species dominated and persisted on all brown rice fumigated with the studied treatments. Brown rice treated with MCPEV was colonized with the least numbers of detected fungal species. The study revealed that compared to control samples, brown rice treated with MCPEV and CRUPV had comparable or slightly higher percentage of non-broken milled rice after storage. However, all the treatments except MCPEV had percentage of non-broken rice lower than the original sample. Improved end product whiteness was only noticeable in MCPEV treated samples.

\section{Acknowledgements}

This research was supported by research grant from the Japanese Society for Promotion of Sciences (JSPS). The authors wish to acknowledge Yamada Apiculture Center, Inc., Japan for their kindness to provide the propolis samples used in this experiment. Further, we acknowledge the technical support of Dr. Eriko Yasunaga and Dr. Satoshi Yoshida of the Biotron institute at Kyushu University in Japan, Dr. Elizabeth Atungulu for technical advice on DNA related analyses and manuscript preparation.

\section{References}

1. Ascherio A. Epidemiologic studies on dietary fats and coronary heart disease. Am J Med. 2002; 113 Suppl 9B:9S-12S.

2. Rice-Evans C.A, Miller N.J, Paganga G. Antioxidant properties of phenolic compounds. Trends Plant Science. 1997; 2(4):152-159. doi:10.1016/S1360-1385 (97)01018-2.

3. Bratter C, Tregel M, Liebenthal C, Volk H. D. Prophylactic effectiveness of propolis for immunostimulation: a clinical pilot study. Forsch Komplementarmed. 1999; 6(5):256-260.

4. Atungulu G, Miura M, Atungulu E, Satou Y, Suzuki K. Activity of gaseous phase steam distilled propolis extracts on peroxidation and hydrolysis of rice lipids. J Food Eng 2007; 80 (3):850-858.

5. Atungulu G, Uchino, T, Tanaka, F, Hamanaka, D. Effect of vapors from fractionated samples of propolis on microbial and oxidation damage of rice during storage. J Food Eng 2008; 88(3):341-352.

6. Silici S, Koc A.N. Comparative study of in vitro methods to analyze the antifungal activity of propolis against yeast isolated from patients with superficial mycoses. Lett Appl Microbiol. 2006; 43(3):318-324.

7. Miura M, Yamauchi F, Ogawa Y, Shibasaki K. Isolation and characterization of proteolipid in defatted soybean meals. Agricultural and Biological Chemistry 1982; 46(6): 1631-1637.

8. Emmons L, Peterson D.M, Paul G.L. Antioxidant capacity of oat (Avena sativa L.) extracts. 2. In vitro antioxidant activity and content of phenolic and tocol antioxidants. J Agric Food Chem. 1999; 47(12):4894- 4898. 
9. Yasumatsu K, Moritaka S, Wada S. Studies on Cereals. Part 4. Volatile carbonyl compounds of cooked rice. Agricultural and Biological Chemistry 1966; 30(5):478-482.

10. Yasumatsu K, Moritaka S, Wada S. Studies on Cereals. Part 5. Stale flavor of stored rice. Agricultural and Biological Chemistry 1966; 30(5):483-486.

11.Zaplin E.S, Liu Q, Li Z, Butardo V.M, Blanchard C.L, Rahman S et al.
Production of high oleic rice grains by suppressing the expression of the OsFAD2-1 gene. Functional Plant biology, 2013; 40(10):996-1004. doi.org/10.1071/FP12301.

12. Anwar F, Anwer T, Mahmood Z. Methodical characterization of rice (Oryza sativa) bran oil from Pakistan. Grasas y Aceites. 2005; 56. Fasc. 2 (2005), 125-134. 